\title{
El estudio del futuro y de la supervivencia del hombre
}

\author{
UN CONSENSO MÁS AMPLIO
}

Estamos viviendo ahora una nueva fase en el desarrollo de las ideas concernientes a la comprensión y a la representación de los procesos globales y planetarios que afectan más directamente la existencia del hombre, y la estimación de las tendencias futuras de tales variables.

La consideración de esta problemática es, en cierta forma, una preocupación humana tan antigua como los primeros intentos en la Grecia Clásica para alcanzar una comprensión filosófica general de la condición humana. Ha estado presente en forma más inmediata en los diversos esfuerzos contemporáneos, desde Spengler y Toynbee hasta un Hain, hacia una comprensión global del proceso histórico y su probable desarrollo futuro. Sin embargo, fue con el Club de Roma, desde su fundación en 1968, bajo el liderazgo de Aurelio Peccei, que se inició la promoción de esfuerzos sistemáticos para alcanzar, de una manera científica y con modelos computarizados, una comprensión y una representación cuantificable de los procesos planetarios complejos que afecten más directamente las sociedades humanas y sus relaciones con la naturaleza.

Si consideramos la breve pero ya rica historia de esta aventura intelectual, desde los esfuerzos iniciales promovidos por el Club de Roma, creo que podemos discernir tres fases. La primera de ellas está marcada por los intentos de Aurelio Peccei y sus colegas iniciales para alcanzar una comprensión total de estos procesos globales como también por sus esfuerzos para descubrir una metodología y una técnica que permitieron la representación cuantitativa de las variables involucradas en ellos, así como de su interacción mutua en un largo período de tiempo, en términos que no pueden ser comprendidos en forma intuitiva. Esta primera fase lleva a los estudios de Jay Forrester y de Dennis Meadows, popularizados luego en el ampliamente conocido libro de éste último "The Limits to Growth", publicado en 1972.

El mensaje central que surge de esta primera fase es en el sentido de que la vieja idea de un progreso general sin límites no puede ser mantenida. Existen límites físicos externos a la expansión del hom. 
bre, determinados por la finitud de nuestro planeta. Estos son, básicamente, de dos tipos. Por un lado, como lo anticipara Malthus, hay límites para el crecimiento de la población, determinados por la capacidad alimenticia del planeta. Por otra parte, en contra de las expectativas de la revolución industrial y del modo de producción capitalista, en general, hay límites para la expansión económica, determinados por la escasez de los recursos no renovables y por la capacidad limitada cle autorregeneración de la biósfera y del ecosistema en general. De ahi, la recomendación de Meadows de alcanzar tan pronto como sea posible un nivel mundial de crecimiento cero, tanto en el plano demográfico como económico.

La segunda fase, en el desarrollo de tales ideas, está marcada por la amplia y violenta discusión de las tesis anteriores, sus supuestos y los modelos que los representan. En último análisis, la crítica más importante se ha referido a cuatro aspectos principales. En primex lugar, se ha enfatizado, siguiendo un camino similar a la crítica de Malthus por parte de Marx, que los puntos de vista de Meadows eran la proyección de una posición sociohistórica reaccionaria. El problema del mundo no es la escasez física sino un sistema de distribución expoliatorio, que concentra la riqueza y el poder en unos pocos grupos centrales de unos pocos países centrales, condenando el resto de la humanidad a una aplastante miseria. Una propuesta de crecimiento cero sólo contribuiria a perpetuar las injusticias in. tolerables de nuestro mundo actual.

Un segundo tipo de crítica concierne al concepto de los límites externos en si mismo, que ha sido despojado de significado por algunos críticos. Dependiendo de cuál régimen y cuáles técnicas de producción se tomen en consideración, el mundo puede tanto ser incapaz de alimentar a la población actual cuanto atender perfectamente las demandas de una sustancialmente mayor, digamos, de treinta mil millones de personas. Las mismas consideraciones se aplican al concepto de recursos no renovables. Existiendo la tecnología y el suministro de energía apropiados como los provenientes de fuentes nucleares prácticamente ilimitadas es posible hacer prácticamente cualquier cosa mediante cualquier cosa.

Un tercer tipo de crítica estaba relacionado con la excesiva agregación de los primeros modelos mundiales. El mundo es un planeta, pero contiene diversas sociedades y regiones. Manejarlo como si fuera una unidad homogénea es totalmente engañoso y trae consigo conclusiones poco representativas.

Finalmente, los críticos han subrayado la completa falta de una dimensión sociopolítica en los modelos de Forrester y Meadows. Todas las catástrofes predichas eran de carácter demográfico y físico, sin ninguna consideración a las reacciones sociopolíticas. 
Siguiendo los lineamientos de estas críticas, la segunda fase en la historia reciente de la futurología condujo a dos nuevos experimentos, entre otros. Uno, emprendido por un grupo de expertos bajo la dirección de Mihajlo Mesarovic y Eduardo Pestel, fue divulgado en un libro publicado en 1975, bajo el título de "Mankind at the Turning Point". El otro, bajo la dirección de Amílcar Herrera y un grupo de expertos de la Fundación Bariloche, de Argentina, fue resumido en un libro que aparecerá con el título de "The Latin American World Model".

Mesarovic-Pestel, aproximadamente siguiendo a Meadows, han construido un modelo matemático más sofisticado, permitiendo la desagregación del mundo en diez regiones y permitiendo en cualquier momento a los operadores del modelo cambiar sus presunciones concernientes al valor de las variables y de los escenarios de acción, escogidos, con el fin de comprobar posibilidades alternativas. El modelo de Bariloche, claramente, es un modelo normativo que está destinado a determinar bajo qué condiciones sería posible, en vista de las tendencias presentes y de las futuras previsibles, asegurar la satisfacción de las necesidades básicas de todos los seres humanos, divididos en cuatro regiones: el mundo desarrollado, América Latina, Africa y Asia. Una de sus interesantes conclusiones es que, con la excepción de Asia, las otras regiones subdesarrolladas serian capaces de alcanzar las metas postuladas desde fines de este siglo hasta principios del próximo, a niveles de ingreso per capita relativamente modestos, siempre que el sistema económico fuera reorientado, dejara de estar basado en el beneficio privado y se orientara hacia el logro de objetivos públicos.

Ahora, como he dicho inicialmente, estamos viviendo en una tercera fase de este proceso. Tal fase se caracteriza, como yo la veo, por una confluencia de ideas, una síntesis de experimentos y un creciente consenso. Tomando en cuenta la contribución de esta primera fase, desde los trabajos iniciales de Aurelio Peccei hasta Denis Meadows; de la segunda fase, desde las críticas de Sussex y Bariloche, hasta el modelo de este último grupo así como el de Mesarovic-Pestel; y considerando la extraordinaria contribución recientemente presentada por Jean Tinbergen ${ }^{2}$ y su grupo, el estimulante informe de la fundación Dag Hammarskjold ${ }^{2}$, y la interesante y

${ }^{1}$ Ver Reviewing the International Order, informe solicitado por el Club de Roma, con el apoyo del gobierno de Holanda, a un distinguido grupo de científicos sociales, coordinados por el profesor Jean Tinbergen.

${ }^{2}$ Ver Otro Desarrollo, Informe Dag Hammarskjold 1975 sobre el desarrollo y la cooperación internacional. 
original contribución presentada por Erwin Leszlow y su grupo, creo que emergen tres importantes conclusiones de este todo.

La primera es de carácter teorético y metodológico. Expone la distinción entre lo que puede llamarse futurologia abstracta y concreta. Una cosa es discutir, en teoría, el problema de los límites externos y otra es discutirlo en un contexto sociohistórico dado. En principio, suponiendo que los otros prerrequisitos serán atendidos en forma conveniente, es técnicamente posible combinando antiguos y nuevos regímenes y medios de producción, producir alimentos suficientes para satisfacer a poblaciones considerablemente mayores de, por ejemplo, 30 mil millones de habitantes. Es igualmente posible usar minerales de baja graduación y otros materiales para producir, con ilimitado suministro de energía, todos los productos industriales requeridos para asegurar una vida de abundancia a todos los miembros de una creciente población, y de controlax en forma conveniente las contaminaciones resultantes del proceso. Otra cosa, sin embargo, es discutir este mismo problema en función de, y partiendo desde, las condiciones y limitaciones presentes, tanto físicas como sociopolíticas y ver cómo, dado el presente estado de cosas, las tendencias actuales y el tiempo disponible, es realisticamente posible prevenir resultados catastróficos y mejorar sustancialmente las condiciones de vida en el futuro previsible.

La segunda conclusión general que se esboza es en el sentido de que, en lo que respecta a un desarrollo realista dentro de las condiciones y limitaciones existentes, no hay duda de que los resultados serán catastróficos, en un futuro no tan distante, si algunas de las tendencias presentes no son controladas oportunamente. Estos resultados catastróficos aparecen indicados claramente, en forma desagregada, en el modelo Mesarovic-Pestel. También emergen claramente del modelo de Bariloche, a pesar de que Ios propósitos de éste son normativos, y no simulativos o predictivos.

La tercera conclusión principal a que se llega es en el sentido de que todavía es posible, viable $\mathrm{y}$ oportuno introducir los cambios necesarios para prevenir las catástrofes y para mejorar sustancialmente las condiciones de vida de la humanidad en general y, particularmente, de las grandes masas destituidas. $Y$ aquí adquiere relieve la contribución del Proyecto Tinbergen, como también los importantes elementos contenidos en el informe de la fundación Dag Hammarskjold y el proyecto Laszlow.

Existen en realidad límites externos, como fue sugerido inicialmente por los que contribuyeron en la primera fase de la futurologia, si bien en una perspectiva diferente. El problema crucial, como fue advertido por los críticos de la segunda fase, es sociopolítico. Aun el crecimiento excesivo de la población - que debe ser con- 
trolado- requiere esencialmente, además de la planificación familiar, un esfuerzo mundial para redistribuir la riqueza y las oportunidades, incluyendo una mejor educación.

\section{LAS SOLUCIONES PROPUESTAS}

Si comparamos cuidadosamente las soluciones propuestas por los más recientes estudios en futurologia, desde Mesarovic-Pestel y Bariloche a Tinbergen y Laszlow, en relación con los desequilibrios mundiales, veremos en último análisis, que éstos corresponden a tres principales grupos de medidas. Hay medidas físicas, concernientes a las nuevas reglas y formas de producción necesarias para ajustar las actividades económicas del hombre a los requerimientos de la preservación del ecosistema. Hay medidas ético-psicológicas, relativas a la necesidad de ajustar los valores y el comportamiento de los individuos y grupos al tipo de metas requeridas por -y compatibles con- las nuevas condiciones de la humanidad. Y hay medidas sociopoliticas, concernientes a la necesidad de lograr, a su debido tiempo, una paz mundial estable y una vida de dignidad y moderada prosperidad - para usar las palabras de Tinbergen- para todos los ciudadanos del mundo, en el contexto de un manejo racional de los recursos mundiales y de un equilibrio entre la población y los medios de sustentación de las diversas regiones del mundo en un momento determinado.

No es el propósito de estas breyes notas el resumir las propuestas específicas presentadas por los estudios bajo consideración, particularmente el conjunto sistemático de recomendaciones de Tinbergen y de Laszlow. Sólo mencionaré que, a mi entender, todas estas propuestas tienen en común tres aspectos fundamentales.

En primer lugar, todas reconocen que los cambios sustanciales requeridos para prevenir resultados catastróficos en el futuro previsible sólo son posibles si hay una profunda y amplia motivación en el mundo, desde ahora en adelantc, para presionar en la clirección conveniente, junto con poderosas fuerzas que cuenten los eventos a través del mismo camino. Esta motivación de acuerclo con todos estos estudios, es esencialmente el autointerés esclarecido de los países y grupos centrales. Y la fuerza que empuja los sucesos en la dirección correcta, de acuerdo también con todos estos estudios, debe ser el autointerés esclarecido del mundo subdesarrollado usando en sus relaciones con el mundo en desarrollo técnicas de negociación colectivas y procedimientos de cartelización para optimizar sus términos de 
intercambio, junto con una mayor cooperación mutua entre los países del Tercer Mundo.

EI segundo aspecto común de todas las soluciones propuestas es la comprobación de que el sistema de las Naciones Unidas es el único que puede ser ajustado para resolver y regular problemas que sobrepasan las fronteras nacionales. Por esto, sin embargo, es indispensable separar las funciones politicas de las Naciones Unidas de sus funciones técnico-administrativas. Las primeras tardarán mucho tiempo en madurar, bajo la forma de un gobierno internacional. Las últimas pueden ser establecidas de inmediato a través del ajuste de los elementos existentes y la creación de nuevas instituciones $y$ autoridades, particularmente para la regulación y manejo de los bienes comunes de la humanidad, tales como los océanos, el espacio, los nuevos recursos y las nuevas fuentes de energia.

El tercer aspecto común de todas las recomendaciones concierne al reconocimiento de la necesidad de un crecimiento fuertemente diferenciado, en términos cuantitativos y cualitativos y en favor del Tercer Mundo, en comparación con los paises desarrollados y, entre los países en desarrollo, relativamente a sus masas pobres y rurales en comparación con los grupos urbanos más favorecidos. Todas estas nuevas políticas de desarrollo, por otra parte, encierran la necesidad de una nueva autoridad y de un nuevo orden monetario internacional, junto con nuevos medios para promover formas ventajosas de transferencia masiva de capitales y tecnología desde los paises y sectores desarrollados a los subdesarrollados.

\section{COMENTARIOS FINALES}

La humanidad subsiste bajo el riesgo permanente e inmediato de aniquilación nuclear. Mientras no se diseñen y adopten nuevas políticas y mecanismos para superar esta situación, a través de nuevas formas cooperativas de control internacional sobre las principales fuentes de violencia, el riesgo de una escalada en cualquier momento, de los conflictos regionales actuales, son extremadamente grandes $y$ peligrosos. Alcanzar las condiciones para una paz mundial estable es un prerrequisito para cualquier programa a largo plazo referente a la supervivencia del hombre.

Supuesto que la hecatombe nuclear pueda ser evitada en las próximas décadas, me parece que existen evidencias suficientes para indicar que la humanidad tratará de prevenir las consecuencias más catastróficas de los actuales desequilibrios del mundo. No es proba. 
ble que el mundo según la hipótesis de Meadows, muera de una hambruna generalizada, ni de la falta total de energía o de materias primas. Ni siquiera de una contaminación generalizada.

Mucho antes de que tales eventos lleguen a asumir las características de una catástrofe mundial generalizada, tenderán a adoptarse políticas y medidas nacionales e internacionales para asegurar a los países y grupos centrales, en detrimento de las masas y países periféricos, el control de los medios necesarios para satisfacer sus necesidades vitales y económicas. Esto tenderá a suceder, al nivel nacional, por la implantación de un despotismo tecnocrático de la izquierda, del tipo stalinista y de la derecha de estilo neofascista. Al nivel internacional esto tenderá a suceder por la consolidación de acuerdos de condominio entre los superpoderes, para asegurar a cada uno de ellos dentro de su respectiva área de hegemonía, los beneficios depredatorios que puedan requerir para su propia conveniencia a expensas de sus respectivas periferias.

En las condiciones culturales y tecnológicas de las primeras civilizaciones, la necesidad de regular el flujo y uso de los ríos para asegurar la estabilidad de las cosechas, llevó a un régimen de organización y producción característico del despotismo oriental. En las condiciones de los tiempos venideros, la necesidad de restablecer un equilibrio mundial viable entre la población y los recursos -si la generación presente no es capaz de adoptar a tiempo las medidas correctivas necesarias- llevará inevitablemente a producir una versión tecnocrática del despotismo oriental, de la cual el stalinismo y el nacismo nos han dado ya una imagen anticipada.

Existe hoy en el mundo la mayor urgencia para comenzar la implantación de programas, básicamente similares al que se propone en el proyecto de Tinbergen. Tales programas representan la última posibilidad para una regulación civilizada, democrática y humanística de nuestros problemas. Si la generación presente no lo hace así, comenzando ahora mismo, la próxima alcanzará algunos de estos objetivos en una forma neostalinista y neofascista.

El problema con que nos enfrentamos de inmediato, en vista de estas consideraciones, es el hecho de que el estímulo y la coordinación que supone tal programa con mucho, supera la capacidad de personas aisladas y de grupos informales, aun de tan distinguidas personas como aquellas reunidas en el Club de Roma. Entre el presente y la movilización mundial de decisiones y medios efectivos para alcanzar los cambios requeridos, es claramente indispensable que haya una mediación organizada, más poderosa y sistemática.

Sólo una institución estable, no oficial y no partidaria, bajo la guía de hombres de la mayor autoridad moral e intelectual, mundial. mente reconocida y provista con recursos financieros y humanos su- 
ESTUDIOS INTERNAGIONALES

ficientes, será capaz de asumir la gigantesca tarea de proveer la maquinaria y las facilidades de infraestructura necesarias para persuadir y movilizar al mundo hacia la búsqueda oportuna de un equilibrio humanístico y democrático. 